\title{
Régionalisme agraire et crédit agricole en \\ Europe Centrale autour de l'année 1930
}

\author{
Sylvain SCHIRMANN
}

\begin{abstract}
As the Great Depression invaded Europe, East-Central countries experienced particular difficulties, owing to their reliance on agriculture. Hence, a series of conferences amongst the latter debated, in 1930, common challenges and potential bases for what we may boldly refer to as a common regional agricultural policy. A broad range of sensitive aspects were brought into discussion, such as overproduction, import rights and free trade, to the mistrust of developed western countries. Albeit the bone of contention may also be found around the institutional dimension of projects centred on agricultural credit, for instance, the aforementioned endeavours were interesting laboratories meant to bring the "two Europes" closer and experiment with ideas of cooperation that manifested themselves under the auspices of Europeanism.
\end{abstract}

Après avoir bénéficié de l'aide américaine à travers le plan Dawes et les investissements qui lui furent consécutifs, l'Europe put envisager la fin de sa reconstruction économique et financière. Les principales monnaies furent progressivement stabilisées et la SDN réfléchit aux principes d'un ordre économique international. La conférence économique de Genève de mai 1927 est, de ce point de vue, essentielle. Elle cherche à imposer deux principes : le démantèlement douanier et la cartellisation, comme moyen de régulation du marché. Si l'on enregistre quelques progrès dans cette direction entre 1927 et 1929 (par exemple le traité de commerce franco-allemand d'août 1927), la question reste posée de sa pertinence pour l'Europe centrale et orientale et plus particulièrement pour les économies pour lesquelles l'agriculture constitue encore un secteur fondamental.

Au moment du plan Briand, ces problèmes restent d'actualité. La crise est déjà présente dans la partie orientale du continent et les difficultés atteignent progressivement l'ouest. La proposition française comporte également une dimension économique forte, et par conséquent il est important d'analyser la position et l'action des pays d'Europe centrale et orientale dans ce contexte. L'importance du secteur agricole dans ces Etats explique pourquoi la question de l'organisation à la fois de la production et du crédit agricole y revêt une importance capitale. Y envisage-t-on une forme de régionalisme, compte tenu de la proximité des problèmes et des situations? Sous quelles formes et avec quels projets ? Quels en sont les résultats?

Une série de conférences agraires entre les pays d'Europe centrale et orientale cherchent à donner forme à ces projets dès 1930 . Force est cependant de constater que progressivement ces perspectives restent illusoires. Elles méritent cependant une réflexion sur les causes de leur échec. 


\section{I- Les conférences agraires ${ }^{1}$}

La conférence agraire qui s'ouvre à Varsovie le 28 août 1930 réunit pratiquement la totalité des pays d'Europe centrale et orientale ${ }^{2}$. Y sont présents les Etats suivants : Pologne, Yougoslavie, Roumanie, Bulgarie, Hongrie, Estonie, Lettonie, Tchécoslovaquie et un observateur finnois. La réunion poursuit plusieurs objectifs. Il s'agit non seulement de constituer un front dans la perspective des conférences d'action économique concertée qui se réunissent à Genève à l'automne 1930, mais également de réfléchir à une meilleure organisation de la production et ainsi penser un espace agricole. Les travaux sont loin d'être vains, car les Etats évoquent une cartellisation de l'agriculture, c'est-à-dire une organisation de la production agricole, sa rationalisation, une réflexion sur une politique des transports, et une ouverture réciproque des marchés. Cela passe par l'octroi réciproque de la clause de la nation la plus favorisée pour le commerce des produits agricoles. Pour éviter une concurrence préjudiciable entre eux, les Etats présents à Varsovie s'engagent à des concertations régulières. Dans l'immédiat, ils instituent dans la capitale polonaise un organisme central commun : un Office d'information et d'harmonisation des questions agricoles. Enfin, ils affichent une forme d'unité politique qui doit déboucher sur une action commune à Genève, et ce en vue de l'obtention d'une convention pour la limitation des restrictions à l'exportation. Le 30 août lorsque la conférence se sépare règne un relatif climat d'optimisme. Selon un responsable polonais, on vient de franchir : « un premier pas vers le bloc agraire des pays de l'Est $»^{3}$.

Deux mois plus tard, le même groupe se retrouve à Bucarest du 18 au 20 octobre 1930. La conférence de Bucarest se situe dans la même veine que celle de Varsovie. A Bucarest, les Etats d'Europe centrale et orientale instaurent entre eux un Comité permanent d'études économiques, qui informe les Etats sur les réalités statistiques de l'agriculture. Ce Comité est invité à travailler avec l'Institut international agricole dont le siège est à Rome. Le travail de réflexion s'y poursuit : chaque pays est

1 On peut se reporter à :

Gilbert NOËL, « La société des Nations et les questions agricoles : géométrie variable et flexibilité », in Sylvain Schirmann (direct.), Organisations internationales et architectures européennes 1929-1939, Metz, Publications du Centre de recherche Histoire et Civilisation de l'université de Metz, 2003, pp. 345-367;

Sylvain Schirmann, Crise, coopération économique et financière entre Etats européens 1929 - 1933, Paris, Cheff, 2000, p. 108 et suiv.

2 Sur cette conférence et les suivantes, l'auteur a bénéficié du dossier "Internationale Konferenzen - April 1929 - Dezember 1930 », de la Reichsbank, Dossier R. 2501/5894, Bundesarchiv, Berlin.

3 D'après un article de la Germania, 31 août 1930, « Eine wirtschaftliche Entente Cordiale des Ostens?». 
déterminé à se lancer au lendemain de la réunion dans une étude sur le crédit agricole. Cette étude est appelée à servir de base à une réunion d'experts qui pourraient promouvoir une attitude commune sur la question. Deux autres points figurent encore à l'ordre du jour et sont largement discutés. Le premier concerne la création d'institutions qui coordonneraient les exportations des Etats d'Europe centrale et orientale ; le second invite les Etats à réfléchir ensemble à un système de traitement préférentiel. La cartellisation de l'agriculture semble ainsi en marche ${ }^{4}$.

La troisième réunion entre le même groupe d'Etats se déroule à Varsovie du 10 au 13 novembre 1930. Elle se penche sur la question du crédit agricole. Ses travaux débouchent sur un Mémorandum consacré au crédit agricole à moyen terme. Celui-ci est destiné à être transmis à la SDN et discuté lors de la conférence d'action économique concertée 5 . Les experts réunis en Pologne pensent que le prêt à moyen terme devrait essentiellement servir à des buts productifs : rationalisation de la production, diminution des coûts, organisation de l'écoulement, amélioration de la qualité. Le prêt serait soit dédié au fonds de roulement des exploitations, soit aux investissements. Les capitaux engagés seraient exigibles dans un délai de 2 à 5 ans et leur utilisation contrôlée par un institut local. Les garanties proviendraient d'hypothèques. Au volet économique de la réorganisation de l'agriculture s'ajoute alors le volet financier. Une impression se dégage progressivement : le front agraire des pays d'Europe centrale et orientale a trouvé en trois mois un terrain d'entente qui pourrait jeter les fondements d'une « politique agricole commune ».

A qui doit-on cette apparente réussite?

\section{II - Les raisons d'un succès}

La Pologne tout d'abord a mis la logistique et la puissance de son administration à la disposition de la conférence : documentation, fonctionnaires. Elle dote le comité d'études de statuts et l'héberge à Varsovie. Les experts polonais lancent les grandes études. Il est certain que sans cette disponibilité matérielle, les travaux n'auraient pas été aussi rapides. Mais il ne faut pas oublier le rôle pionnier d'un trio. La Yougoslavie, la Roumanie et la Hongrie se sont en effet retrouvées le 24 juillet 1930 à Bucarest pour tenter de répondre en commun au questionnaire élaboré par la SDN pour les conférences d'action économique concertée et envisager ensemble le principe du régime préférentiel pour leurs productions agricoles ${ }^{6}$. Cette réunion est suivie d'une autre début août 1930 à Sinaia entre Roumains et Yougoslaves, au cours de laquelle les deux Etats conviennent de la nécessité d'un modus vivendi

4 L'auteur a pu consulter les archives de la SDN à Genève à ce sujet. Sur cette conférence, Archives SDN, document $2^{\mathrm{e}}$ conf./AEC/9, Protocole de la conférence des experts agricoles du Centre et du Sud-Est de l'Europe qui s'est tenue à Bucarest du 18 au 20 octobre 1930.

5 Archives de la SDN, SDN, document $n^{\circ}$ C.149.M.48.1931, II. B., p. 256 et suiv.

6 Bundesarchiv, Berlin, Deutsche Reichsbank, R. 2501/5894. 
portant sur la concession d'avantages mutuels dans les échanges. Ils expriment leur volonté d'éviter une concurrence à l'exportation qui leur serait préjudiciable, et souhaitent réfléchir à une éventuelle création d'organes communs. Les deux décident également d'inviter la Tchécoslovaquie aux rencontres ultérieures et s'engagent à communiquer le résultat de leurs travaux et leur calendrier éventuel aux autres Etats d'Europe centrale. Ces différentes initiatives permettent sur les questions agricoles à un régionalisme infra européen de prendre corps ${ }^{7}$. Celui-ci répond de surcroît pleinement au contexte d'alors.

La crise vient d'abord à l'esprit. Les pays d'Europe centrale et orientale qui exportent essentiellement des produits agricoles se heurtent de plus en plus à la surproduction mondiale, à la baisse des prix et à la politique protectionniste des pays qui constituent leurs marchés habituels. S'y ajoutent souvent des problèmes de paiement, qui débouchent sur des situations sociales problématiques. Mais les Etats réagissent également à une initiative de Genève : l'action économique concertée. Ces réunions se comprennent en effet à la lumière de la politique menée par la SDN. Son comité économique souhaite mettre en route la trêve douanière décidée à la conférence économique internationale de 1927. A l'assemblée de septembre 1929, les Etats se rallient au principe d'une conférence d'action économique concertée qui a vocation à aller vers la baisse du protectionnisme et à organiser la production et les marchés, aussi bien industriels qu'agricoles ${ }^{8}$. En février - mars 1930, une réunion préliminaire de cette conférence se tient à Genève pour entendre les différentes positions des Etats. Les Etats d'Europe centrale et orientale sont prêts à accepter l'idée d'une trêve douanière si un certain nombre de dérogations ou de possibilités d'action leur sont données. Ils envisagent ainsi un abaissement du protectionnisme qui frappe les productions agricoles comme l'exprime par exemple De Nickl, le représentant hongrois à cette réunion : «La Hongrie exporte des produits agricoles et elle constate que nombre d'Etats qui devraient, par nécessité, importer de tels produits, pratiquent un protectionnisme exagéré, dont ils attendent le relèvement de leur agriculture, mais qui par ses répercussions mêmes ébranlent la vie économique de la Hongrie $»^{9}$.

D'autres encore envisagent purement et simplement des mesures de rétorsion. Le délégué yougoslave le laisse entendre clairement : il faut « inaugurer dans nos Etats un protectionnisme industriel $»^{10}$. Polonais et Roumains défendent quant à eux l'idée de contracter des ententes économiques régionales. Le ministre roumain

7 Bundesarchiv, Berlin, Deutsche Reichsbank, R.2501/5894, Abschluss der Konferenz von Sinaia.

8 Antoine Fleury, «Un sursaut anti-protectionniste dans le contexte de la crise de 1929 : le projet d'une trêve douanière multilatérale», Relations internationales, n 39,1984

9 SDN, document $\mathrm{n}^{\circ}$ C. 222. M. 109. 1930, II, Intervention septième séance, 20 février 1930, p. 110.

10 SDN, document $n^{\circ}$ C. 222. M. 109. 1930. II, Intervention huitième séance, 20 février 1930, p. 115. 
Madgearu en fixe les contours. Il s'agit « d'accepter une dérogation à la clause de la nation la plus favorisée dans ce sens qu'un traitement préférentiel soit appliqué aux Etats qui seront partie à ces accords [régionaux]. » Ces regroupements locaux, élaborés en fonction de structures économiques comparables ou d'intérêts communs sont à ses yeux « une première étape dans la voie de l'établissement de l'unité économique européenne $\rangle^{11}$. Dans le débat sur l'organisation de l'Europe, si le projet Briand s'intéresse à une construction qui intègre l'ensemble de l'Europe, d'autres propositions s'appuient sur le régionalisme infra européen comme élément de la mise en place d'une unité européenne, notamment une unité économique. Il suffit de se reporter à la vision exprimée par le belge Paul Hymans, le 5 septembre 1929 ou par l'ouvrage de Delaisi sur les deux Europes, pour comprendre que la résolution de 1927 sur le libre-échange appliquée sans discernement pouvait conduire à la catastrophe. Pour ces personnalités, il s'agissait de donner corps à des marchés communs régionaux, seuls viables à leurs yeux. C'est à partir de ces unions douanières qu'il fallait penser progressivement l'union économique de l'Europe ${ }^{12}$.

Les résolutions de la conférence préliminaire consacrent, le 24 mars 1930, le principe de la trêve douanière jusqu'au $1^{\mathrm{er}}$ avril 1931 et admettent des exceptions pour les produits agricoles. Une dernière disposition prévoit un programme de négociations ultérieures. Pour le lancer, la SDN envisage d'envoyer un questionnaire aux gouvernements ; celui-ci constituerait la base des discussions qu'il conviendrait de mener avant la fin de la trêve en avril 1931. Or, ce questionnaire comporte des interrogations quant à la limitation des excédents agricoles, à la réduction des droits sur les céréales, à l'élaboration de conventions plurilatérales, bref contient des chapitres qui intéressent les pays d'Europe centrale et orientale ${ }^{13}$. C'est pour préparer ces discussions prévues pour novembre 1930, que les pays d'Europe centrale et orientale imaginent le régionalisme présenté dans les lignes précédentes. La conférence d'action économique concertée se réunit en effet en 2 sessions : du 17 au 28 novembre 1930 (1 $1^{\text {ere }}$ session), du 16 au 18 mars 1931 pour la $2^{\text {ème }}$ session. Elle est saisie d'une demande déposée conjointement par la Yougoslavie, la Roumanie, la Hongrie, la Pologne et la Bulgarie. Leur texte insiste sur trois points:

- le traitement préférentiel, notamment pour les céréales;

- la consolidation de droits d'importation de produits agricoles à un niveau raisonnable;

11 SDN, document $n^{\circ}$ C. 222. M. 109. 1930, II, Intervention cinquième séance, 19 février 1930, p. 92.

12 Sur le régionalisme, voir par exemple, Eric Bussiere, «L'Organisation économique de la SDN et la naissance du régionalisme économique en Europe », in Relations internationales, $\mathrm{n}^{\circ} 75,1993$, pp. 301-313.

13 Voir Résumé des travaux mensuels de la Société des Nations, Volume X, n 3, mars 1930, pp. 89-93. 
- la liberté complète du commerce des produits en provenance de l'Europe de l'Est et du Centre.

L'Europe centrale conformément aux échanges des mois précédents relatés ci-dessus s'exprime ainsi en faveur d'une ouverture des frontières des Etats développés de l'Ouest européen. Cette ouverture devrait lui permettre d'affronter la crise dans de meilleures conditions. La contrepartie serait constituée par un effort commun de restructuration agricole destiné à moderniser le monde rural ${ }^{14}$. Cette " politique agricole commune » que mèneraient ces Etats serait ainsi un facteur de leur développement et sécuriserait cette partie de l'Europe. Objectifs économiques et politiques allaient de pair.

\section{III - L'échec du régionalisme agraire centre}

européen et la question du crédit agricole

Lors des conférences d'action économique concertée l'Europe centrale et orientale se heurte néanmoins à la réticence des pays industriels de l'Ouest européen. L'attitude de ces derniers provoque l'irritation de leurs partenaires centreeuropéens. Manoilesco, le représentant roumain, l'exprime en ces termes : « $\mathrm{Si}$ l'Amérique du Nord avait conquis pour ses produits industriels l'Orient de l'Europe, comme l'Amérique du Sud a conquis l'Occident pour les produits agricoles, l'idée de préférence et de protectionnisme européen serait peut-être née, non pas dans l'Orient, mais dans l'Occident de l'Europe $\gg^{15}$. Les réticences occidentales ouvrent également la voie à l'Allemagne, prête à des négociations bilatérales.

D'autres questions sont abordées au cours de cette session : sur la méthode faut-il accorder des préférences douanières ou ne vaut-il pas mieux développer les capacités de concurrence des pays d'Europe centrale et orientale pour les amener à rivaliser efficacement avec les pays d'Outre-Mer? Polonais et tchèques souhaitent la mise en place d'une organisation technique et financière qui favorise la mutation agricole. En tout état de cause, pour les Etats d'Europe centrale et orientale, la priorité reste l'équilibre de cette partie du continent, avec un système de préférences sans contrepartie, car ce mode de fonctionnement est déjà un avantage pour l'ensemble du continent et tient lieu de réciprocité. Fierlinger, le représentant tchécoslovaque, le dit sans réserve : " insister sur un régime préférentiel bilatéral... signifierait que l'on envisage le problème dans un esprit mercantile et que l'on veut accentuer la règle du donnant-donnant, ce qui dans ce cas s'appliquerait à des situations tout à fait inégales ». La réciprocité figerait économiquement le continent et ne règlerait en rien la question agricole pour les Etats danubiens. Le

14 SDN, Document $2^{\mathrm{e}}$ conférence/AEC/ 10, Propositions soumises par Manoilesco au nom de la Pologne, de la Yougoslavie, de la Roumanie, de la Bulgarie et de la Hongrie, le 19 novembre 1930 .

15 SDN, Document n C. 149. M. 48. 1931. II. B., 19 novembre 1930, p. 69 
système préférentiel est pour ces Etats l'amorce de la coopération sur le continent européen, économique d'abord, politique ensuite. Il faut donc - les experts polonais insistent là-dessus - une organisation de la production et du commerce des produits agricoles, à la fois à l'échelle infra régionale et à la fois à l'échelle continentale. Ils posent à nouveau la question du crédit agricole. Seul, ce dernier pointretient l'attention et donne lieu à décision. Pour le reste tout est en suspens. Comment empêcher alors certains Etats d'envisager les préférences dorénavant par la voie du bilatéralisme ? En mars 1931, selon les mots même de Colijn, le néerlandais qui préside la Conférence d'action économique concertée, « les efforts déployés en vue de mettre en application les recommandations de la conférence économique mondiale ont abouti à un échec complet ». Il signifie par la même occasion l'échec de l'action multilatérale en Europe ${ }^{16}$.

Reste finalement la question du crédit agricole qui pourrait constituer l'ultime bouée de secours pour la partie orientale du continent. Doter l'Europe centrale et orientale d'institutions modernes de crédit agricole comblerait indiscutablement une lacune. Le projet est ancien car la question d'un organisme international de crédit agricole est étudiée dès 1926 à l'Institut international d'Agriculture à Rome. La crise relance ce projet souhaité par les Etats d'Europe centrale et orientale à partir de 1930. Deux banques privées qui s'étaient préoccupées de ces questions, la Compagnie centrale de Prêts fonciers d'Amsterdam et le Crédit foncier international de Bâle n'ont pas les ressources suffisantes et ne peuvent donc courir de grands risques dans cette partie de l'Europe. C'est en janvier 1931, entre les deux sessions de la conférence d'action économique concertée que la Commission d'études pour l'Union européenne, créée dans la foulée du mémorandum Briand de 1930, adopte une résolution sur l'institution d'un Crédit agricole international financé par les Etats détenteurs de capitaux. Elle instaure un comité chargé de coopérer avec le Comité financier de la SDN et souhaite qu'un projet complet soit soumis au Conseil de la SDN pour sa session de mai $1931^{17}$.

C'est donc dans l'urgence entre février et avril 1931 qu'est imaginé un Institut international, une Société internationale de crédit hypothécaire agricole, dont les premiers statuts sont rédigés par Eugène Renard, sous-gouverneur du Crédit foncier de France. C'est un organisme d'utilité publique, mais de droit privé, contrôlé par la SDN, dont le siège devrait être fixé à Paris. Ses opérations permettraient de rembourser les prêts usuraires et modifieraient les cultures de prêt. Son capital (50 millions de dollars) serait souscrit par des personnes physiques ou morales privées.

16 Sur l'ensemble des Conférences d'action économique concertée, lire Sylvain Schirmann, Crise, coopération économique... op. cité, pp. 83-105.

17 Sur la question du crédit agricole, Schaeffer Patrick J., « Les illusions de la coopération financière européenne au début de la crise des années 1930 : l'exemple de la société internationale de crédit hypothécaire agricole » in Sylvain Schirmann (direct.), Organisations internationales et ... op. cité, pp. 367-386. 
Pour offrir des garanties solides, en bout de chaine le crédit serait garanti par des hypothèques. Celles-ci seront détenues par un institut national, seul organisme à qui l'on prêtera. A lui de voir par quel système local il passera pour acheminer l'argent vers le secteur agricole. Mais ce dossier est également éminemment politique. Paris et Londres approuvent cette perspective pour des raisons différentes. Le gouvernement britannique, conscient du fait que les capitaux seraient essentiellement français en espère un avantage pour la Livre, dans la mesure où la pression qui pèse sur elle se relâcherait. Mais l'opinion publique anglaise se désintéresse du projet. Paris soutient la perspective, dans la mesure où les actionnaires représenteront une partie du capital ce qui ne peut qu'avantager la France. Quant à l'Allemagne, en pleine crise de la tentative d'Anschluss économique (mars 1931), elle manifeste une méfiance envers la Société, qu'elle considère comme un moyen de pénétration économique française en Europe centrale et orientale. Les textes sont malgré tout adoptés à l'unanimité le 14 mai 1931 et Paris renonce au siège qui est fixé à Genève. Le Conseil de la SDN approuve la création et les statuts du nouvel organisme le 21 mai 1931. Le 5 juin, Eugène Regard est porté à la tête du nouvel organisme et le travail, en attendant la ratification, peut commencer. Au 30 septembre 1931, on a déjà réuni 31 millions de francs suisses!

Mais la Société ne voit pas le jour. Paris traîne et la ratification n'intervient pas. Les autorités françaises doivent faire face à l'opposition des chambres d'agriculture et du Quai d'Orsay. Pour les responsables de la diplomatie française, la place faite à la France, qui apporte l'essentiel des capitaux, est insuffisante. Elle doit en effet, à travers la SDN et les statuts de l'organisme, partager la gouvernance avec d'autres Etats, qui exercent ainsi à ses yeux un contrôle sur des fonds français. Le Royaume-Uni n'en fait pas non plus une priorité. Il a choisi d'autres préférences : elles sont impériales, et non centre européennes. L'Allemagne préfère la logique du bilatéralisme, tout comme l'Italie et, dès l'automne 1931, une série d'accords de ce type desserre quelque peu l'étau. Cela leur permet de faire rentrer petit à petit ces Etats de l'Europe centrale et orientale dans leur orbite. L'Allemagne, par exemple, signe en juin et juillet 1931 trois accords avec la Bulgarie, la Roumanie et la Hongrie. Elle leur accorde des réductions de droits sur les céréales. L'Italie réserve à ces Etats (Yougoslavie, Roumanie, Hongrie) des ristournes et des abaissements de taxes. La conférence du Bloc agraire réunie à Varsovie le 27 août 1932 réclame quant à elle l'entrée en fonction de la Société de crédit agricole, pratiquement un an après sa constitution. Sans succès. Il en est de même pour le fonds de revalorisation des céréales et le fonds de soutien monétaire imaginé depuis le début de l'année 1932. Ces projets ne sont d'ailleurs plus adaptés à l'Europe centrale et orientale, car c'est un plan d'une autre ampleur qu'il faut pour relancer l'économie dans cet espace.

$\mathrm{Au}$ total, le débat a été fécond. La crise a permis le rapprochement dans cette partie de l'Europe de pays révisionnistes et de pays satisfaits par l'ordre versaillais. 
Les solutions discutées laissaient entrevoir l'émergence d'une politique agricole régionale, qui avec l'aide des puissances de l'ouest européen, pouvait peut-être stabiliser le continent dans un contexte économique difficile, mais également dans un climat de débats prometteurs fruit des initiatives de la SDN depuis 1927 et du plan Briand. Force est de reconnaître que l'instrumentalisation de la crise par les puissances a eu raison de ces projets. Celles-ci préfèrent développer avec leurs voisins de l'Est des rapports fondés sur le clientélisme. Ces rapports favorisent l'entrée des Etats de l'Europe centrale et orientale dans l'orbite des puissances révisionnistes, qui ont bien davantage à leur offrir dans le cadre des accords bilatéraux, que la France et le Royaume-Uni, timides et recroquevillés sur leurs égoïsmes nationaux. Incapables de renoncer à des vues étroites, elles ne savent donner corps à des projets ambitieux auxquels les Etats d'Europe centrale ont commencé à réfléchir au tournant des années 1930. Si la responsabilité des puissances est largement engagée pour comprendre l'échec de ces tentatives, il ne faut pas oublier non plus de méditer sur la solidarité effective entre des pays qui n'hésitèrent pas dès 1931 à se lancer dans des accords bilatéraux qui font fi des réflexions entamées entre eux en 1930. De telles dispositions minent la confiance et rendent difficile la poursuite d'une politique agricole régionale. Faut-il cependant ne garder en mémoire que cet échec aux perspectives tragiques?

Les desseins esquissés par les pays d'Europe centrale sont à bien des égards prometteurs. L'européisme qui les caractérise fourmille de réflexions sur la nécessité de lier les deux Europes, interroge la méthodologie de la construction d'une Europe unie. Il vise à élaborer une politique agricole régionale, s'intéresse à la régulation du marché agricole et à l'organisation de la production de manière telle à garantir des ressources aux agriculteurs. Au-delà, c'est une ébauche d'un système de préférences que les desseins tentent d'élaborer, reposant également sur la solidarité entre l'Est et l'Ouest du continent. Cette solidarité comporte à travers les projets de crédit agricole un volet financier. A regarder cela, il nous faut constater que certaines de ces idées refont surface dans le cadre de la construction européenne qui part à l'Ouest dans les années 1950. Elles y ont été portées par des hommes mêlés aux discussions d'alors (Jules Gauthier, Andreas Hermes...), par des organisations (l'Institut international d'agriculture). Au-delà de l'échec dans le contexte des années 1930, ces projets restent un laboratoire d'idées en matière d'européisme! 